\title{
An Ultrafast Hydrogel Photocrosslinking Method for Direct Laser Bioprinting
}

Zongiie Wang ${ }^{1}$, Xian $\mathrm{Jin}^{1}$, Ru Dai ${ }^{1,2}$, Jonathan F. Holzman ${ }^{1}$, and Keekyoung Kim ${ }^{1, *}$

${ }^{1}$ School of Engineering, University of British Columbia, Kelowna, BC, V1V 1V7,

Canada

${ }^{2}$ West China Hospital, West China Medical School, Sichuan University, Chengdu, 610041, China

*Corresponding author: Dr. Keekyoung Kim, keekyoung.kim@ubc.ca 


\begin{abstract}
Photocrosslinking is a widely-used method to generate cell-laden hydrogels for tissue engineering. At present, it usually takes more than 30 seconds to crosslink hydrogels using UV illumination, and this delay makes it more likely that damage will occur in the DNA. With this in mind, we introduce an ultrafast photocrosslinking method using a low-cost blue laser diode. Experimental results show that a hydrogel with a diameter of 8 mm can be crosslinked using this process within 10 seconds with over $90 \%$ cell viability. Moreover, it is shown that the laser can be focused for the implementation of bioprinting. A microscale cell-laden microtube was successfully fabricated with this laser-based system, demonstrating its feasibility for bioprinting.
\end{abstract}




\section{Introduction}

Photocrosslinkable hydrogels have been used in a number of tissue engineering applications, including cell differentiation, injectable tissue generation, bioprinting, and organ-on-a-chip. ${ }^{1-9}$ Photocrosslinking has many advantages over conventional physicalor chemical-crosslinking methods. The advantages include spatial and temporal control in the polymerization process, fast crosslinking rates (several seconds to a few minutes) at room temperature, as well as minimal heat production. ${ }^{4}$ To achieve the fast photocrosslinking of hydrogels, many researchers have applied expensive high-intensity light sources, such as the OmniCure Series 2000 (Excelitas Technologies, Waltham, USA), with wavelengths spanning the 100 to $400 \mathrm{~nm}$ ultraviolet (UV) spectrum..$^{9,10}$ However, it is still difficult to crosslink cell-laden hydrogels within 30 seconds even with these UV light sources. (The crosslinking time is often several minutes when using a standard handheld UV lights.) At the same time, it has been proposed that exposure to UV light can induce DNA damage ${ }^{11-13}$ or the cancerization of cells. ${ }^{14,15}$ Thus, it is highly desirable to find a crosslinking method for the polymerization of cell-laden hydrogels that is both fast and not reliant upon UV radiation.

In this work, we present a simple, ultrafast crosslinking method to create cell-laden hydrogels using a low-cost (off-the-shelf) laser diode at a wavelength of $405 \mathrm{~nm}$. The developed method is shown to complete the crosslinking process within 10 seconds, and the cell viability right after crosslinking is found to be higher than $90 \%$. Cells could also 
attach and proliferate inside the laser-crosslinked hydrogels, demonstrating that the laser-based crosslinking method has a high potential for tissue engineering. A hydrogel pattern is printed for this study to demonstrate that the proposed direct laser crosslinking method.

To achieve a fast crosslinking speed, the applied light source should have a sufficiently high intensity at the absorption peak of the photoinitiator. Moreover, the light source should ideally have a wavelength that is higher than the UV-B band, i.e., higher than $315 \mathrm{~nm}$, because radiation in the UV-B band has been reported to induce serious DNA damage and apoptosis. ${ }^{11,13}$ Given these considerations, a laser is an ideal source for crosslinking, as a laser can output the desired high-intensity radiation with a narrow linewidth (at the absorption peak of the photoinitiator). Moreover, the advent of GaN-based laser diodes has introduced a new generation of inexpensive and compact light sources with wavelengths in the proximity of $405 \mathrm{~nm}$. Such laser diodes can match the absorption peaks of many biocompatible photoinitiators.

There are many biocompatible photoinitiators for cell encapsulation, such as 2-hydroxy-4- (2-hydroxyethoxy)-2-methylpropiophenone (Irgacure 2959). ${ }^{16}$ However, it is difficult to directly apply this photoinitiator to laser crosslinking, since the absorption peak of Irgacure 2959 is at a wavelength of approximately $260 \mathrm{~nm} \cdot{ }^{17}$ Irgacure 2959 under such 265-nm irradiation can crosslink hydrogels very fast, i.e., within a few seconds, but this UV-B irradiation can readily induce DNA damage and apoptosis of cells, as stated 
above. ${ }^{11,13}$ It has been reported that Irgacure 2959 also functions under UV-A irradiation, at a wavelength of approximately $365 \mathrm{~nm}$, which is safer in comparison to UV-B irradiation, but it takes a long time to complete the crosslinking process ${ }^{18}$ due to the relatively low molar extinction coefficient (being equivalent to the absorption coefficient) of the long-wave UV-A light. ${ }^{19}$ According to our investigation, Irgacure 2959 is not activated at a wavelength of $405 \mathrm{~nm}$, and this result is in agreement with previous reports that the molar extinction coefficient of Irgacure 2959 reaches zero at wavelengths higher than $370 \mathrm{~nm} .{ }^{19}$ Thus, it is concluded that Irgacure 2959 cannot be used for the laser-based photocrosslinking at a wavelength of $405 \mathrm{~nm}$.

The photoinitiator, 2,2'-azobis[2-methyl-n-(2-hydroxyethyl)propionamide] (VA-086), was recently introduced as a promising photoinitiator for tissue engineering. ${ }^{20}$ Its absorption peak is at approximately $385 \mathrm{~nm}$. This is in the long-wave UV-A range, which allows for activation by laser diodes with short crosslinking times. ${ }^{21}$ It is also worth noting that VA-086 is more biocompatible than Irgacure 2959, which contributes to high cell viability after crosslinking. ${ }^{21,22}$ The work presented here shows that the hydrogel with VA-086 can be crosslinked with illumination by a 405-nm laser diode (Fig. 1A shows the crosslinking mechanism). Such a system can be accelerated, by applying illumination for 10 seconds over an 8-mm-diameter beam spot, to achieve the so-called "ultrafast" crosslinking. 


\section{Materials and Methods}

\section{Light source}

The critical component of the ultrafast crosslinking method is the laser diode. A 405-nm blue laser diode with a peak power of $300 \mathrm{~mW}$ (Armlaser, San Francisco, CA, USA) was employed for this. The laser diode was mounted $10 \mathrm{~cm}$ above a petri dish. A DC power supply (CPS-3205, Gopher Technology, Guangzhou, China) was connected to the laser diode, and its voltage was adjusted to control the current supplied to the laser diode-which ultimately controlled the power being emitted by the laser diode. A schematic and photograph of the crosslinking system are given in Figs. $1 \mathrm{~B}$ and $1 \mathrm{C}$, respectively. The power of the laser was measured by an optical power meter (Model 1916R, Newport, Irvine, CA, USA). Three laser powers were applied, over a circular beam spot with a diameter of $8 \mathrm{~mm}$, yielding laser intensities of $1 \mathrm{~mW} / \mathrm{mm}^{2}, 2 \mathrm{~mW} / \mathrm{mm}^{2}$, and $5 \mathrm{~mW} / \mathrm{mm}^{2}$, respectively.

\section{Hydrogel synthesis}

The hydrogel prepolymer solution for the experiment is based on a gelatin methacrylate (GelMA) hydrogel. The GelMA was synthesized using a previously reported method. ${ }^{16}$ Briefly, we dissolved gelatin (from porcine skin) in dimethyl sulfoxide (DMSO) solvent at $50^{\circ} \mathrm{C}$ with stirring to have a $10 \% \mathrm{w} / \mathrm{v}$ gelatin in the DMSO solution. Then, 4-dimethylaminopyridine (DMAP) and glycidyl methacrylate were added to the solution 
to form a mixture with $0.6 \% \mathrm{w} / \mathrm{v}$ DMAP and $7.4 \% \mathrm{v} / \mathrm{v}$ glycidyl methacrylate. The mixture was stirred for two days at $50^{\circ} \mathrm{C}$. Subsequently, the mixture was dialyzed against reverse osmosis (RO) water at room temperature for a week. The RO water was changed twice a day. After dialysis, the GelMA sample was freeze-dried via lyophilization. For the experiments, a photocrosslinkable GelMA prepolymer solution was prepared by dissolving 10\% w/v freeze-dried GelMA and 1\% w/v VA-086 photoinitiator (Wako Chemical, Osaka, Japan) in phosphate buffered saline (PBS). Unless stated otherwise, all materials were purchased from Sigma-Aldrich, St. Louis, MO, USA.

\section{Bioprinting system setup}

To build a bioprinting system, we integrated the laser diode with a spherical lens $(f=50$ $\mathrm{mm}$ ) to achieve a focused laser spot with a diameter of approximately $400 \mu \mathrm{m}$. A one-axis linear actuator (L12R, Firgelli Tech., Victoria, BC, Canada) was employed as a stage on which to move the petri dish. The setup of the modified system is shown in Figs. $1 \mathrm{D}$ and 1E. With the bioprinting system setup, the focused laser spot can be used to selectively crosslink the hydrogel prepolymer solution during motion of the stage. In this way, the laser-based platform can print cell-encapsulated microtube patterns in one direction.

Before bioprinting, the $10 \% \mathrm{w} / \mathrm{v}$ GelMA solution was mixed with NIH 3 T3 fibroblast cells $\left(8 \times 10^{6}\right.$ cells $\left./ \mathrm{mL}\right)$. Then, $2 \mathrm{~mL}$ of GelMA solution was pipetted and dispensed onto 
the petri dish (being $3.5 \mathrm{~cm}$ in diameter) to form a thin layer of GelMA solution. The laser diode was then switched on to generate the focused laser spot. At the same time, the linear actuator was used to apply motion at a constant speed of $3 \mathrm{~mm} / \mathrm{s}$. With the movement of the stage, the laser spot crosslinked a line of hydrogel microtubes on the petri dish. After bioprinting, the uncrosslinked hydrogel was removed. The microtubes were washed twice with PBS. Subsequently, fresh media were added to the petri dish and the drop was cultured inside the incubator.

Cell culture, encapsulation, and viability assessment

NIH $3 \mathrm{~T} 3$ fibroblast cells were cultured at $37^{\circ} \mathrm{C}$ with $5 \% \mathrm{CO}_{2}$. The cell media consists of Dulbecco's Modified Eagle Medium (DMEM) with $10 \% \mathrm{v} / \mathrm{v}$ fetal bovine serum and 1\% $\mathrm{v} / \mathrm{v}$ penicillin streptomycin. All the materials were purchased from ThermoFisher Scientific, Carlsbad, CA, USA. The cell medium was changed every three days.

Experiments were carried out to investigate the biocompatibility of the laser-based crosslinking within various parameters. Before crosslinking, the $10 \%$ GelMA prepolymer solution was mixed with NIH 3 T3 fibroblast cells $\left(8 \times 10^{6}\right.$ cells $\left./ \mathrm{mL}\right)$. Then, $100 \mu \mathrm{L}$ drops of the $10 \%$ GelMA prepolymer solution were pipetted onto the petri dish. The drops containing cells were then illuminated and crosslinked by the laser beam. After crosslinking, the drops were washed twice with PBS. Subsequently, fresh media were added to the petri dish and the cell-laden GelMA drops were cultured inside the incubator. 
In order to verify the biocompatibility of the laser crosslinking, we checked the cell viability and proliferation at day 0 , day 2, and day 4 using a LIVE/DEAD assay (Biotium, Hayward, CA, USA). Samples were washed three times with PBS and treated with the assay for 30 minutes. The images of the assayed samples were taken by a confocal fluorescence microscope (FV1000, Olympus, Tokyo, Japan).

To analyze the cell viability, images taken with the microscope were converted to a 16-bit gray value format, and the cell numbers were counted manually using cell counter plugin provided by FIJI ImageJ (NIH, Bethesda, MD, USA). In the end, the cell viability was calculated with the following formula:

$$
\text { Cell viability }=\frac{\text { Number of live cells }}{\text { Number of all cells }}
$$

In addition, we compared the cell density (being the total number of total cells per $\mathrm{mm}^{2}$ ) at day 0 , day 2 , and day 4 to quantify the proliferation of cells. The cell density was calculated as follows:

$$
\text { Cell density }=\frac{\text { Number of live cells in the field of view of the microscope }}{\text { Area of the field of view of the microscope }} .
$$

\section{Statistical analysis}

A one-way analysis of variance (ANOVA analysis) function in MATLAB 2014b (MathWorks, Natick, MA, USA) was used to statistically analyze all the data. The results are presented as the average \pm standard deviation. The sampling size (the time of replicates) was three. 


\section{Results and Discussion}

Laser-based cell-laden hydrogel crosslinking

Occhetta et al. reported that the VA-086-based photocrosslinking process generated nitrogen gas during crosslinking, making the hydrogels cloudy. ${ }^{20}$ Thus, to find the optimal crosslinking time, we illuminated the GelMA prepolymer solution with the laser until the bubble generation reached a saturation point-which was assumed to be the point at which the GelMA achieves a fully-crosslinked state. As shown in Fig. 2A, the hydrogel was free of bubbles before crosslinking. With the increase of the crosslinking time, bubble generation inside GelMA hydrgoel continually increased, until it reached the fully-crosslinked state at which bubble generation stopped. We found that the minimum times needed for crosslinking 10\% GelMA hydrogel with the 1\% VA-086 photoinitiator at $50 \mathrm{~mW}, 100 \mathrm{~mW}$, and $250 \mathrm{~mW}$ laser powers were 30 seconds, 20 seconds, and 10 seconds, respectively. Based on the minimal crosslinking time, we defined three combinations of laser powers and crosslinking time, $50 \mathrm{~mW}-30$ seconds, $100 \mathrm{~mW}-20$ seconds, and $250 \mathrm{~mW}-10$ seconds, to conduct cell encapsulation experiments to examine the cell viability after crosslinking.

The cell encapsulation results are presented in Fig. 2B. The green dots (Calcein AM) represent live cells, and the red dots (Ethidium Homodimer III) represent dead cells. It can be seen that at day 0 (right after crosslinking), most of the cells were still alive. After two days' culturing, some of the cells attached and started to elongate inside the 
microenvironment of the hydrogels. At day 4, more cells were elongated compared to day 2, however, the number of dead cells increased slightly. As mentioned above, VA-086-based photocrosslinking has the disadvantage that it generates bubbles inside the crosslinked hydrogel. Fig. 2C shows that there were many bubbles inside the hydrogels at day 0 . However, the bubbles disappeared as the cells grew and the hydrogel degraded. At day 4 , there were a negligible number of bubbles inside the hydrogel. This observation is similar to the results reported by Occhetta et al. ${ }^{21}$

Fig. 3A presents the results of the cell viability analysis. It can be concluded that the ultrafast crosslinking process has high biocompatibility. At day 0 and day 2, the viabilities of all samples were higher than $90 \%$, while the viability at day 4 was significantly decreased. The decreased viability at day 4 can be explained by the relatively high cell density inside the hydrogel after four days of culturing. The cell proliferation analysis, seen in Fig. 3B, reveals that the cell density continued to increase up to day 4, at which point it may become difficult for some cells to obtain enough nutrition to live. ${ }^{23}$ However, even at day 4 , the cell viability was greater than $80 \%$. As shown in Fig. 3A, it was found that the p-value between the viabilities of $50 \mathrm{~mW}$ and 250 $\mathrm{mW}$ at day 4 was 0.059 , which is not considered to be statistically significant. Thus, the cell viability was not affected to a great extent by the chosen combinations of crosslinking time and laser power. However, in terms of practicality, it can be seen that the higher power laser diode $(250 \mathrm{~mW})$ is seen to offer improved performance by way of 
its shorter crosslinking time.

\section{Bioprinting application}

The high cell viability of the encapsulated cells and the short crosslinking time led us to employ the developed laser-based photocrosslinking method for bioprinting applications. With the bioprinting system setup illustrated in Figs. 1D and E, the focused laser spot can be used to selectively crosslink the hydrogel prepolymer solution during motion of the stage. In this way, the laser-based platform can print cell-encapsulated microtube patterns in one direction.

The phase contrast and fluorescence images of a hydrogel microtube crosslinked by the laser spot are presented in Figs. 4A and 4B, respectively. The cells attached and grew inside the microtube after three days of culturing, with a cell viability of over $90 \%$. Three-dimensional images of bioprinted microtubes were visualized by BioView3D (Version 0.1.18, Center for BioImage Informatics, University of California, Santa Barbara, CA, USA). The constructed three-dimensional image in Fig. 4C shows that the laser-based crosslinking method is a promising solution for bioprinting. It is also worth noting that the use of a focused laser spot reduces the generation of bubbles. According to our observation, with a laser spot having a $400 \mu \mathrm{m}$ diameter, there are no bubbles generated in the microtube after printing. Even smaller laser spot diameters can be applied by focusing via microscope objectives, and this can enable laser-based 
bioprinting and competitive cell patterning on a microscale.

It is important to note that the demonstrated laser-based bioprinting technique differs

from existing laser-assisted bioprinting techniques. ${ }^{24-26}$ Laser-assisted bioprinting typically utilizes an energy-absorbing layer, in response to the stimulus of lasers, with the cells suspended below the absorbing layer. Once the small area of the absorbing layer is illuminated by the laser, a high-pressure bubble is generated, propelling the suspended cells below that area. This forms a droplet for collection by the receiving board. ${ }^{27,28} \mathrm{In}$ this study, by contrast, the laser directly illuminates and crosslinks the desired area to pattern the hydrogel scaffolds. There is no droplet formation process and no supporting layer. Thus, we have named our technique "direct laser bioprinting." More research will be carried out in the near future to investigate the fabrication of complex three-dimensional cell-laden scaffolds using the direct laser bioprinting.

\section{Conclusion}

We have presented an ultrafast photocrosslinking method using a low-cost laser diode and a VA-086 photoinitiator. The developed technique was able to crosslink the hydrogel within 10 seconds, and the cell viability after patterning was over $90 \%$. By implementing this technique with a focusing lens, we established a bioprinting system for spatially-resolved photocrosslinking. We found that microscale ultrafast crosslinking avoided bubble generation during crosslinking. The successful fabrication of a $400 \mu \mathrm{m}$ 
hydrogel microtube demonstrated the potential of this direct laser bioprinting system.

\section{Acknowledgement}

This work was supported by the Natural Sciences and Engineering Research Council of Canada (NSERC) Discovery Grant (RGPIN-2014-04010).

\section{References}

1 A. Khademhosseini, R. Langer, J. Borenstein and J. P. Vacanti, Proc. Natl. Acad. Sci. U. S. A., 2006, 103, 2480-7.

2 P. Zorlutuna, N. E. Vrana and A. Khademhosseini, IEEE Rev. Biomed. Eng., 2013, 6, 47-62.

3 Z. Wang, B. Calpe, J. Zerdani, Y. Lee, J. Oh, H. Bae, A. Khademhosseini and K. Kim, Biotechnol. Bioeng., 2015, In press.

$4 \quad$ K. T. Nguyen and J. L. West, Biomaterials, 2002, 23, 4307-4314.

5 Z. Wang, R. Abdulla, B. Parker, R. Samanipour, S. Ghosh and K. Kim, Biofabrication, 2015, 7, 045009 .

6 K. C. Hribar, P. Soman, J. Warner, P. Chung and S. Chen, Lab Chip, 2014, 14, 268-275.

7 Z. Wang, R. Samanipour, K. Koo and K. Kim, Sensors Mater, 2015, 27, 487-506.

8 Z. Wang, R. Samanipour and K. Kim, in Biomedical Engineering: Frontier Research and Converging Technologies, Springer International Publishing, 2015, pp. 209-233.

9 C. Cha, J. Oh, K. Kim, Y. Qiu, M. Joh, S. R. Shin, X. Wang, G. Camci-unal, K. Wan, R. Liao and A. Khademhosseini, Biomacromolecules, 2014, 15, 283-290.

10 J. Jung and J. Oh, Biomicrofluidics, 2014, 8, 036503.

11 J. Cadet, E. Sage and T. Douki, Mutat. Res. Mol. Mech. Mutagen., 2005, 571, 3-17.

12 R. P. Sinha and D. P. Häder, Photochem. Photobiol. Sci., 2002, 1, 225-236.

13 D. Kulms, E. Zeise, B. Pöppelmann and T. Schwarz, Oncogene, 2002, 21, 5844-51.

14 B. K. Armstrong and A. Kricker, J. Photochem. Photobiol. B Biol., 2001, 63, 8-18.

15 F. R. De Gruijl, H. J. Van Kranen and L. H. F. Mullenders, J. Photochem. Photobiol. B Biol., 2001, 63, 19-27. 
J. W. Nichol, S. T. Koshy, H. Bae, C. M. Hwang, S. Yamanlar and A. Khademhosseini, Biomaterials, 2010, 31, 5536-44.

17 W. A. Green, Industrial Photoinitiators: A Technical Guide, CRC Press, Boca Raton, 2010.

J. R. Tse and A. J. Engler, Curr. Protoc. Cell Biol., 2010, 1-16.

Y. H. Roh, J. H. Park, J. J. Ye, J. E. Lee and D. Luo, ChemPhysChem, 2012, 13, 2517-2521.

P. Occhetta, N. Sadr, F. Piraino, a Redaelli, M. Moretti and M. Rasponi, Biofabrication, 2013, 5, 035002 .

21 P. Occhetta, R. Visone, L. Russo, L. Cipolla, M. Moretti and M. Rasponi, J. Biomed. Mater. Res. Part A, 2015, 103, 2109-2117.

22 A. D. Rouillard, C. M. Berglund, J. Y. Lee, W. J. Polacheck, Y. Tsui, L. J. Bonassar and B. J. Kirby, Tissue Eng. Part C Methods, 2011, 17, 173-179.

23 S. Ozturk and W.-S. Hu, Cell Culture Technology for Pharmaceutical and Cell-Based Therapies, CRC Press, Boca Raton, 2005.

24 F. Guillemot, a Souquet, S. Catros, B. Guillotin, J. Lopez, M. Faucon, B. Pippenger, R. Bareille, M. Rémy, S. Bellance, P. Chabassier, J. C. Fricain and J. Amédée, Acta Biomater, 2010, 6, 2494 500 .

25 B. Guillotin, A. Souquet, S. Catros, M. Duocastella, B. Pippenger, S. Bellance, R. Bareille, M. Rémy, L. Bordenave, J. Amédée and F. Guillemot, Biomaterials, 2010, 31, 7250-6.

26 V. Keriquel, F. Guillemot, I. Arnault, B. Guillotin, S. Miraux, J. Amédée, J.-C. Fricain and S. Catros, Biofabrication, 2010, 2, 014101.

27 S. V Murphy and A. Atala, Nat. Biotechnol., 2014, 32, 773-785.

28 C. Mandrycky, Z. Wang, K. Kim and D.-H. Kim, Biotechnol. Adv., 2015, 1-13. 


\section{Figures}

A<smiles>C=C(C)C(=O)NC1CCCCC(CC)NC1=O</smiles>
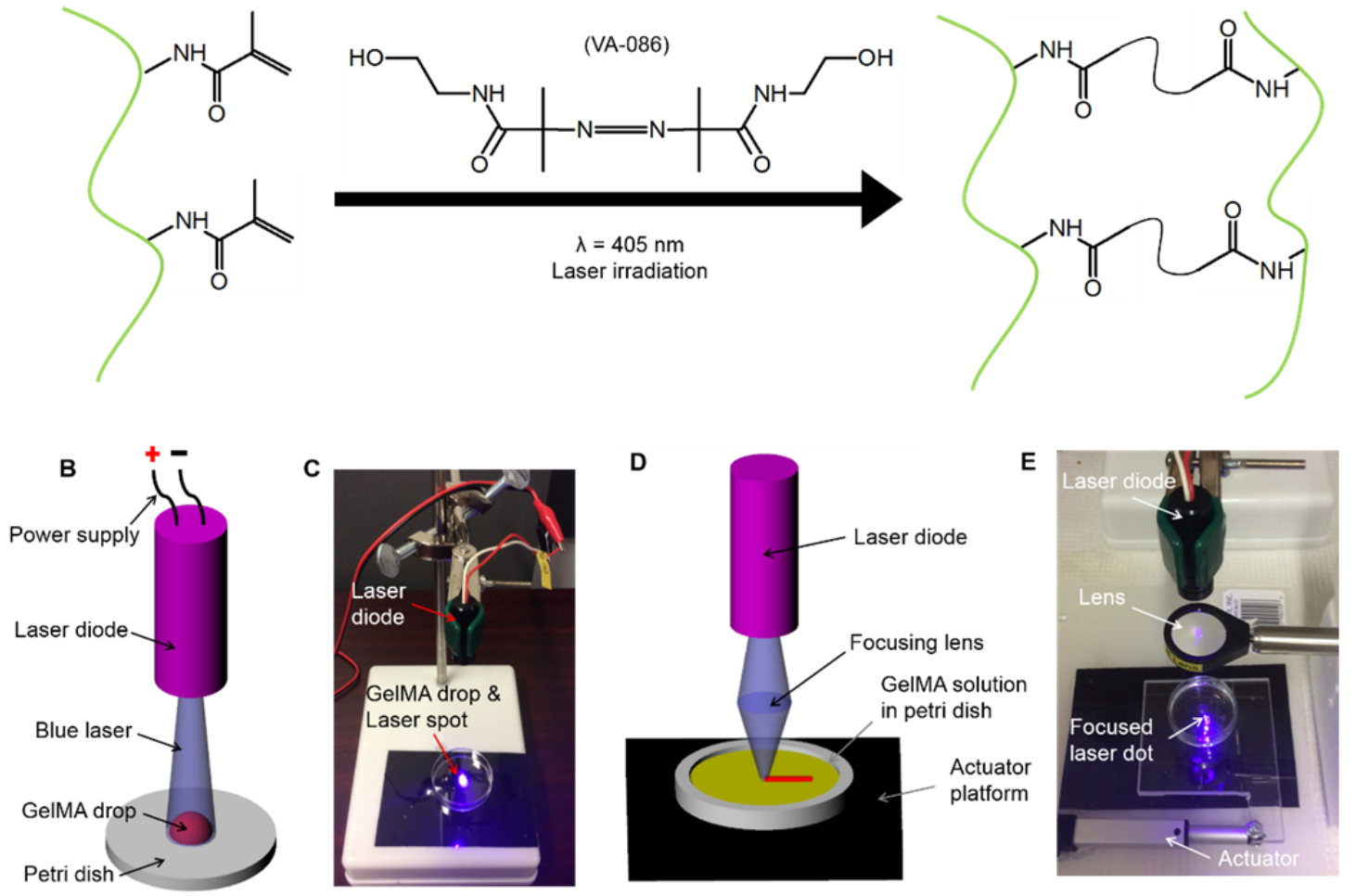

Fig. 1. Laser-based crosslinking and bioprinting system set-up. (A) Laser-based photocrosslinking mechanism. GelMA hydrogel network was formed in the presence of VA-086 photoinitiator and the blue $(\lambda=405 \mathrm{~nm})$ laser irradiation. (B) A schematic of the simple laser crosslinking system. (C) A photograph of the crosslinking system setup. (D) A schematic of the direct laser patterning system shows that the focused laser spot prints a hydrogel microtube by the movement of a linear actuator. (E) A photograph of the laser printing system setup. 

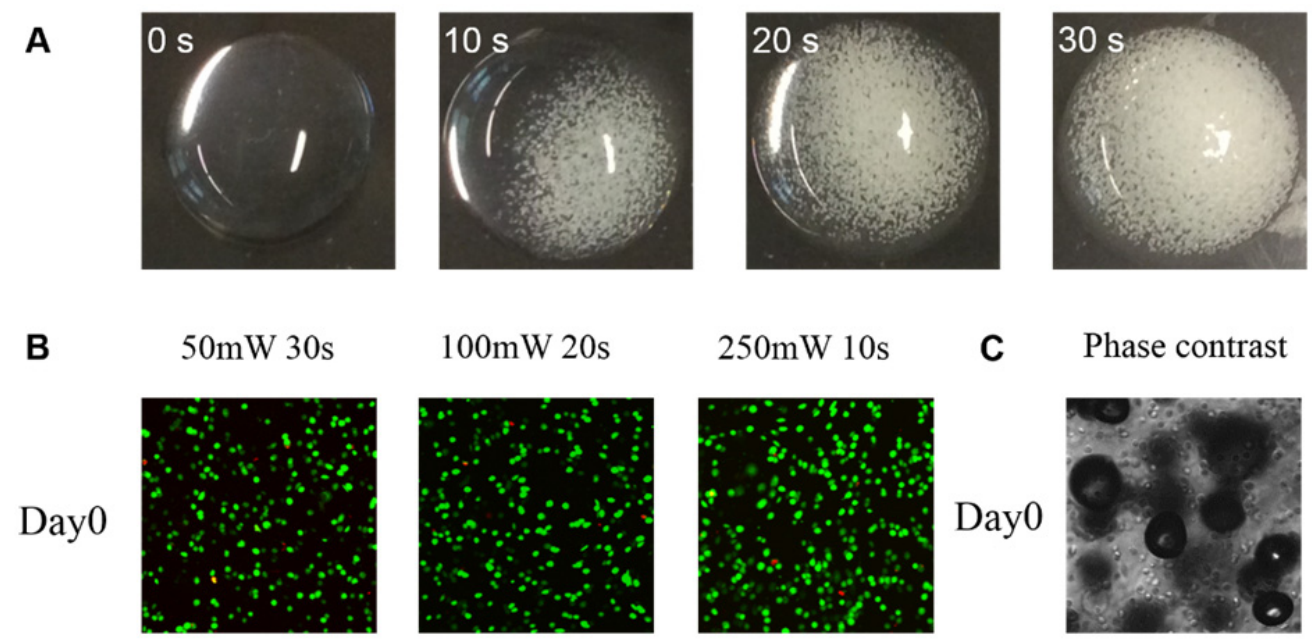

$100 \mathrm{~mW} 20 \mathrm{~s}$

$250 \mathrm{~mW} \mathrm{10s}$

C

Phase contrast
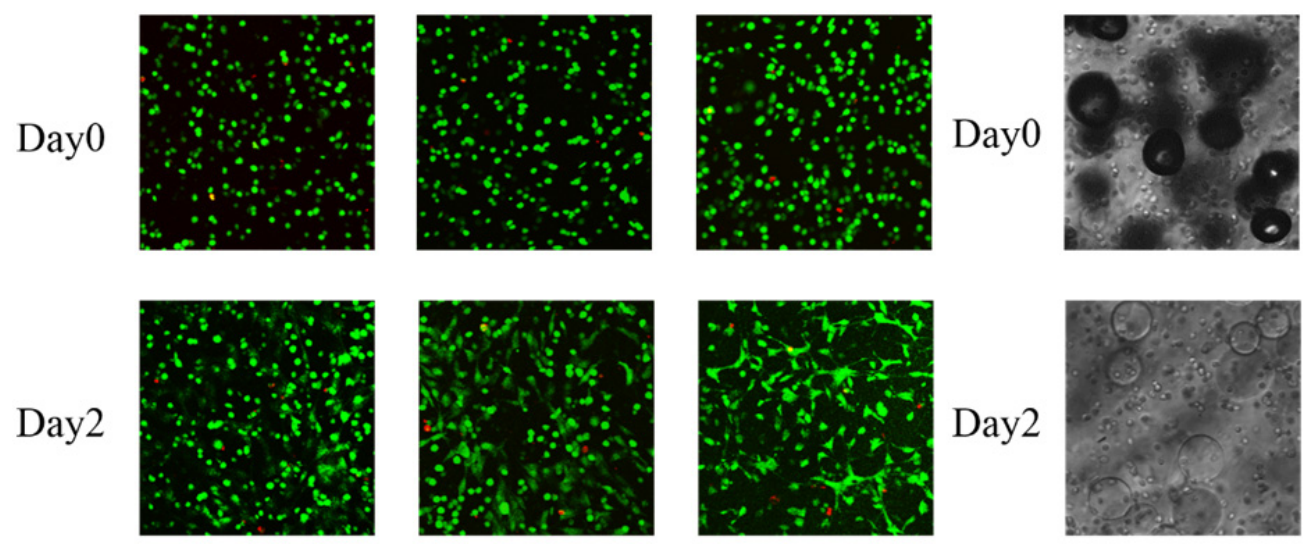

\section{Day2}
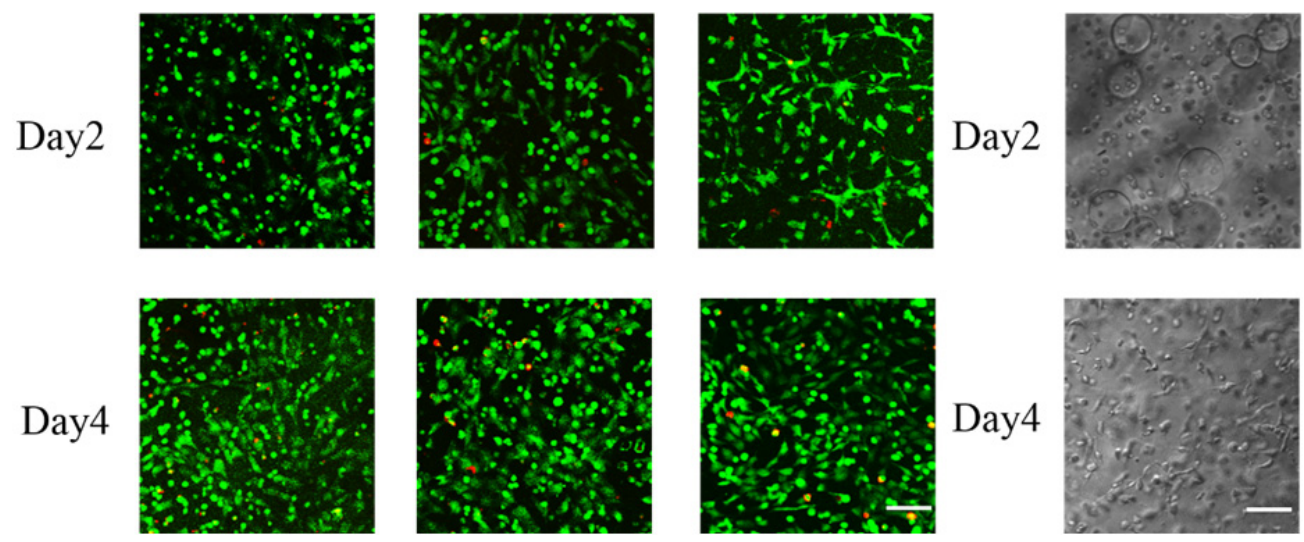

Day4

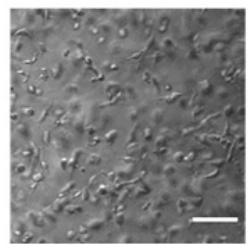

Fig. 2. Pictures of the crosslinked hydrogel drops. (A) Pictures of hydrogel with different crosslinking time. The power of the laser was $50 \mathrm{~mW}$. (B) Confocal fluorescence images of the crosslinked cell-laden GelMA at day 0 , day 2, and day 4. Scale bar $=100 \mu \mathrm{m}$. (C) Phase contrast images of the crosslinked cell-laden GelMA with $250 \mathrm{~mW}-10$ seconds at day 0 , day 2 , and day 4 . Scale bar $=100 \mu \mathrm{m}$. 


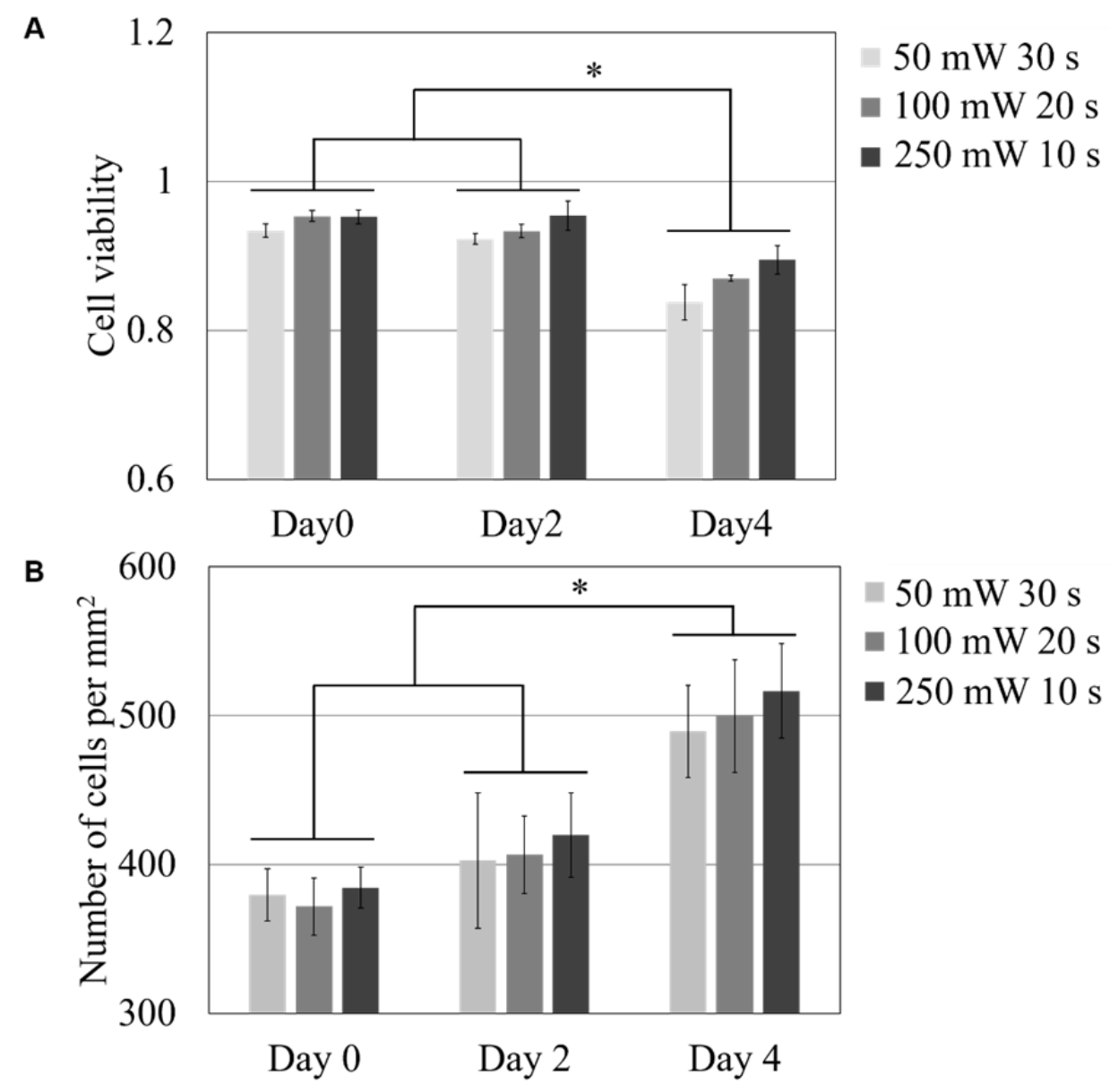

Fig. 3. Statistial analysis of the cell-laden GelMA. (A) Viability, and (B) Cell density of the cells cultured inside the crosslinked hydrogel drops. Error bars indicate the standard deviation $(\mathrm{n}=3, * \mathrm{p}<0.05)$. 

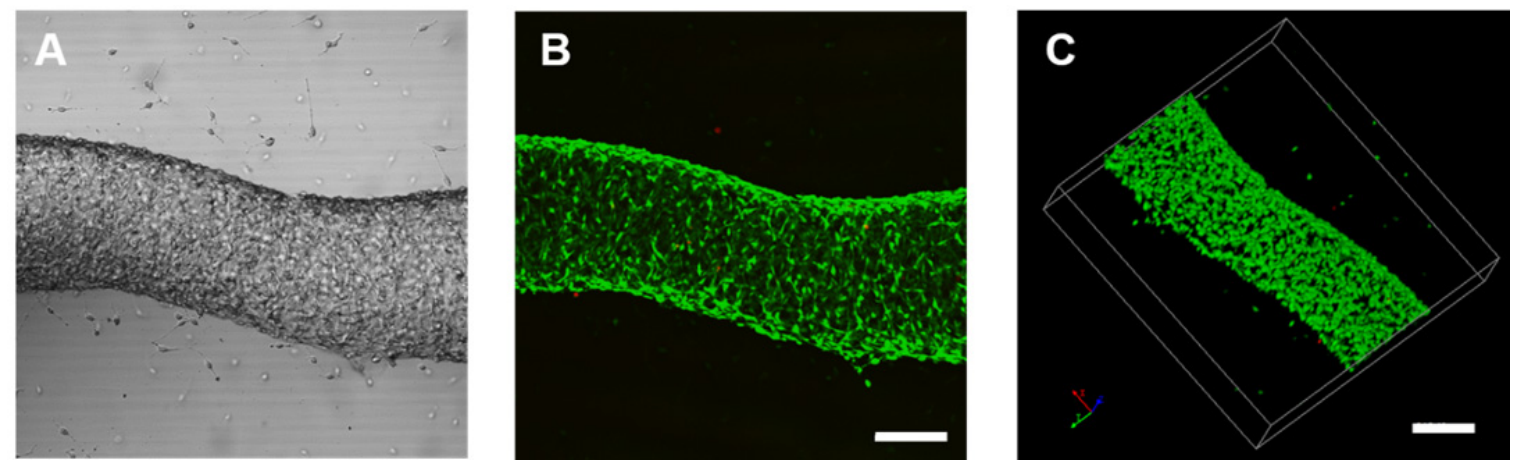

Fig. 4. Direct laser bioprinted microtube sample. (A) A phase contrast image, (B) fluorescence live/dead assyed image (Scale bar $=200 \mu \mathrm{m}$ ), and (C) 3-D reconstructed image of the printed GelMA microtube on day $3($ Scale bar $=215.5 \mu \mathrm{m})$. 